HEINRICH-HEINE-INSTITUT DÜSSELDORF

ARCHIV - BIBLIOTHEK - MUSEUM

HERAUSGEGEBEN VON JOSEPH A. KRUSE

BAND 6 
Susanne Cramer

\section{Die Musikalien}

des Düsseldorfer Musikvereins

(1801-1929)

Katalog

Verlag J. B. Metzler

Stuttgart - Weimar 


\section{HEINRICH-HEINE-INSTITUT DÜSSELDORF \\ ARCHIV · BIBLIOTHEK · MUSEUM \\ HERAUSGEGEBEN VON JOSEPH A. KRUSE \\ BAND 6}

Die Deutsche Bibliothek - CIP-Einheitsaufnahme

Cramer, Susanne:

Die Musikalien des Düsseldorfer Musikvereins (1801-1929) :

Katalog / Susanne Cramer.

- Stuttgart ; Weimar : Metzler, 1996

(Archiv, Bibliothek, Museum / Heinrich-Heine-Institut Düsseldorf ; Bd. 6)

ISBN 978-3-476-01412-2

NE: HST; Heinrich-Heine-Institut <Düsseldorf>: Archiv, Bibliothek, Mueseum

ISBN 978-3-476-01412-2

ISBN 978-3-476-03637-7 (eBook)

DOI 10.1007/978-3-476-03637-7

Dieses Werk einschließlich aller seiner Teile ist urheberrechtlich geschützt. Jede Verwertung außerhalb der engen Grenzen des Urheberrechtsgesetzes ist ohne Zustimmung des Verlages unzulässig und strafbar.

Das gilt insbesondere für Vervielfältigungen, Übersetzungen, Mikroverfilmungen und die Einspeicherung und Verarbeitung in elektronischen Systemen.

(C) 1996 Springer-Verlag GmbH Deutschland

Ursprünglich erschienen bei J. B. Metzlersche Verlagsbuchhandlung

und Carl Ernst Poeschel Verlag GmbH in Stuttgart 1996 


\section{Inhaltsverzeichnis}

$\begin{array}{ll}\text { Vorwort } & 7\end{array}$

Zum Geleit $\quad 9$

1. Einleitung

1.1 Generelle Merkmale des Notenbestandes des Städtischen Musikvereins Düsseldorf $\quad 11$

1.2 Das historische Umfeld 12

1.3 Das musikalische Repertoire $\quad 18$

1.4 Quellentypische Merkmale 24

2. Hinweise zur Verwendung des Katalogs

2.1 Vorbemerkung 28

2.2 Art und Numerierung der Eintragungen 29

2.3 Einordnung der gedruckten Notenausgaben $\quad 30$

2.4 Ordnung der Eintragungen mit demselben Sachtitel 32

2.5 Hinweise zur bibliographischen Beschreibung innerhalb der Formalkatalogisierung 32

2.6 Weitere Erschließung der Notenausgaben 33

2.7 Erfassung des handschriftlichen Notenmaterials

3. Siglen und Abkürzungen $\quad 36$

4. Katalog 39

Verzeichnis der Stempel des Musikvereins Düsseldorf 360

$\begin{array}{ll}\text { Literaturverzeichnis } & 361\end{array}$

Werkregister $\quad 365$

Textincipts der Lieder, Gesänge und geistlichen Vokalwerke 379

$\begin{array}{ll}\text { Peronenregister } & 384\end{array}$ 


\section{Vorwort}

Alle Bücher haben ihre Geschichte, alle Bibliotheken und ihre Handschriftensammlungen auch. Diese Geschichte prägt den Bestand und die Sammeltradition, verursacht Veränderungen, Beschränkungen und damit gleichzeitig einhergehende vertiefende Erweiterungen. Das HeinrichHeine-Institut ist als Literaturarchiv und Literaturmuseum unter dem Namen der poetischen Galionsfigur Heine die Nachfolgeeinrichtung der Neueren Handschriftenabteilung der ehemaligen Landes- und Stadtbibliothek Düsseldorf (1770-1970), deren Buchbestand, mittelalterliche Handschriften und frühe Drucke als Dauerleihgabe von der Stadt Düsseldorf an die heutige Bibliothek der Heinrich-Heine-Universität Düsseldorf übertragen worden sind. Düsseldorf und die niederrheinisch-bergische Region stehen im Mittelpunkt der Sammlungen des Heinrich-Heine-Instituts vom Barock bis zur Gegenwart aus den Gebieten Literatur, Kunst, Musik und Wissenschaft. Heine und Schumann, die Düsseldorfer Malerschule und das kulturelle Leben mehrerer Jahrhunderte sind in Archiv, Bibliothek und Museum des Heinrich-Heine-Instituts dokumentiert.

Ein glücklicher Zufall hat dem Heinrich-Heine-Institut auch den einmaligen Altbestand aus dem Notenarchiv des Städtischen Musikvereins Düsseldorf als Depositum beschert. Dafür ist dem Musikverein und als Vermittler Herrn Dr. Bernhard R. Appel von der Schumann-Forschungsstelle Düsseldorf aufrichtig zu danken. Der Deutschen Forschungsgemeinschaft ist Dank zu sagen für Ihre Fördermittel, durch die es Frau Dr. Susanne Cramer ermöglicht wurde, das Notenarchiv des Musikvereins durch den vorliegenden Katalog aufzuarbeiten. Ihr, aber auch ihrem akademischen Lehrer, Herrn Prof. Dr. Klaus Wolfgang Niemöller vom Musikwissenschaftlichen Institut der Universität Köln, der mit Umsicht das Engagement der Bearbeiterin für die Erschließung begleitet hat, ist besonders herzlich und aufrichtig Dank zu sagen.

Joseph A. Kruse 


\section{Zum Geleit}

Der hier vorgelegte Katalog über den umfangreichen Altbestand aus dem Notenarchiv des Städtischen Musikvereins Düsseldorf hat nicht nur für die regionale Musikgeschichte des 19. Jahrhunderts, namentlich der Niederrheinischen Musikfeste, einen hohen Wert, den neue authentische Quellen vermitteln, sondern er überschreitet in seiner Singularität in zweierlei Hinsicht die bisher praktizierten Standards von musikhistorischen Quellenkatalogen. Zunächst wird hiermit ein entschiedener Schritt in Richtung einer Erweiterung des bisherigen zeitlichen Rahmens älterer Quellendokumentation getan. Das Internationale Quellenlexikon der Musik (RISM) begrenzt den Erfassungszeitraum bis 1800. Mit diesem Katalog wird nun dem in der Musikforschung vielfach mit guten Argumenten vorgetragenen Desiderat Rechnung getragen, auch die musikalischen Quellen des 19. Jahrhunderts müßten systematisch und spezifisch erfaßt, aufgearbeitet und erschlossen werden. Nachdem u. a. die gehaltvolle Publikationsreihe "Studien zur Musikgeschichte des 19. Jahrhunderts" der Thyssen-Stiftung seit 1965 auch dieses Jahrhundert in seiner geistig-künstlerischen Vielfalt in das Blickfeld der internationalen musikwissenschaftlichen Forschung gerückt hatte, nachdem nunmehr im Rahmen des Akademie-Programms der Konferenz der Akademien der Wissenschaften neue kritische Gesamtausgaben von bedeutenden Komponisten des 19. Jahrhunderts, wie C. M. von Weber, Fr. Schubert, F. Mendelssohn, R. Schumann und J. Brahms in Angriff genommen worden sind, gewinnt das Erscheinen dieses Katalogs eine Art paradigmatischen Charakter.

Als ein von der Bibliothekskommission der Deutschen Forschungsgemeinschaft gefördertes Programm ist zu Recht von einem "Spezialbestand" die Rede. Eines der herausragenden Merkmale ist die Heterogenität und Vielschichtigkeit des Notenmaterials, die sich aus fachwissenschaftlicher Sicht wegen des hohen Ranges der Quellen zu einer methodischen Herausforderung entwickelte. Originalausgaben, wertvolle Erst- und Frühdrucke von Partituren, Klavierauszügen, Chor- und Orchester-Stimmen, dazu aber auch in erheblichem Umfang handschriftliches Stimmenmaterial ergeben in dieser Gemengelage methodische Probleme der Katalogisierung von der Titelaufnahme bis zur Datierung. Der grundlegende Gebrauchscharakter verbindet sich wegen der zeitlichen Nähe zu wichtigen Urauffuihrungen mit einer hier spezifisch beglaubigten Werküberlieferung etwa für Mendelssohn und Schumann. So war es auch unerläßlich, die wichtigen handschriftlichen Eintragungen zu erfassen, denn sie betreffen nicht nur signifikante auffiihrungspraktische Hinweise, sondern auch konkrete Korrekturen und Zusätze bedeutender Komponisten zu den von ihnen selbst aufgeführten Werken. Dieses Erfassen der handschriftlichen Zusätze ist eine Erweiterung der for- 
malisierten Kataloge, was letztlich zwingend weitere Recherchen herausforderte, die zu einer ersten Stufe inhaltlicher Aufarbeitung führt; weitere Forschungen können und müssen sich anschließen.

Die musikphilologischen und dokumentarischen Aspekte des Notenarchivs, das in aufregenden Etappen erst quasi wiederentdeckt werden mußte, verweisen in repräsentativer Weise auf ein umfassendes Repertoire einer aktiven bürgerlichen Musikkultur des Chorwesens. Die weltlichen und gesitlichen Gattungen von den oratorischen Werken bis hin zu den zeitbedingten Gebrauchskompositionen der 2. Jahrhunderthälfte erfahren hier eine Anschaulichkeit und Präsenz, die - so ist zu hoffen - auf die weitere Forschung anregend und befruchtend wirkt.

Klaus Wolfgang Niemöller

\section{Danksagung}

Allen voran möchte ich Herrn Dr. Appel und Herrn Dr. Wendt von der Schumann-Forschungsstelle Düsseldorf herzlich für zahlreiche Hilfeleistungen danken. Herr Dr. Appel stellte mir bereitwillig wichtige Quellen aus dem Bestand der Forschungsstelle zur Verfügung. Herr Dr. Wendt stand mir tatkräftig bei der technischen Arbeit mit Computer, Drucker und Software zur Seite. Beide Herren gaben mir überdies unverzichtbare Hinweise für die Datierung und Beschreibung der Musikalien, insbesondere jener zu den Werken von Robert Schumann, sowie für die Identifizierung der Schumannschen Eintragungen. Frau Annette Müller danke ich herzlich dafür, daß sie mir ausgehend von den Vorarbeiten zu ihrer Dissertation über die Kopisten Robert Schumanns wertvolle Informationen zu den Düsseldorfer Kopisten und den handschriftlichen Quellen der Schumannschen Werke zukommen ließ.

Mein besonderer Dank gilt den Herren Professoren Niemöller und Kruse für ihre betreuende Tätigkeit und wichtige Denkanstöße. Dem Heinrich-Heine-Institut und insbesondere Frau Hermstrüwer danke ich außerdem für Quellen, Materialien und Anregungen zu der Düsseldorfer Kulturgeschichte im 19. Jahrhundert.

Herrn Micheal Cooper danke ich dafür, daß er mich auf Eintragungen Mendelssohns in dem Auffürungsmaterial aufmerksam machte und mir außerdem eine Liste der von Mendelssohn in Düsseldorf aufgeführten Werke zur Verfügung stellte. Aufrichtig danke ich auch den Herren Wehnert und Hellmundt von der Leipziger Mendelssohn Ausgabe bei ihrer Hilfe zur Identifizierung der Mendelssohnschen Handschrift.

Nicht zuletzt danke ich Herrn Dr. Hardeck vom Hochschulbibliothekszentrum Köln dafür, daß er mir bei vielen Fragen und Problemen bei der Formalkatalogisierung bereitwillig Auskunft gab. 